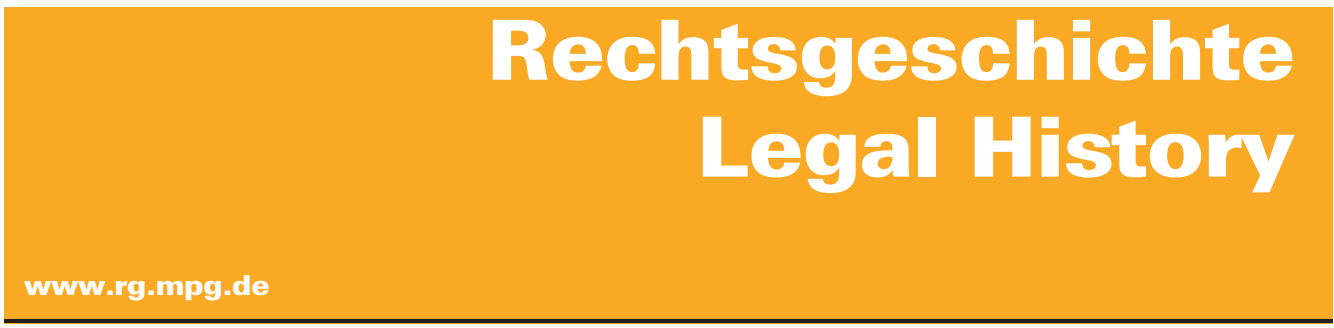

http://www.rg-rechtsgeschichte.de/rg23

Rg $22015 \quad 325-329$

Zitiervorschlag: Rechtsgeschichte - Legal History Rg 23 (2015)

http://dx.doi.org/10.12946/rg23/325-329

\title{
Johannes Liebrecht
}

Teile ohne Ganzes 
präsentiert sich die Studie als eine Analyse eines spezifischen regionalen Rechtsraumes. Einerseits gelingt es Ströhmer die makrohistorischen Befunde im »kleinen Raum« am Beispiel des Oberamtes Dringenberg mit Erfolg zu verifizieren, andererseits gerät das Gesamtsystem kaum in den Blickwinkel. Der Autor unterlässt es die Ergebnisse in einen größeren Zusammenhang einzuordnen, die Makroebene (Germania Sacra) wird allenfalls gestreift. Gerade die lokale Engführung und die ausschließliche Konzentration auf lokale Quellen auf allen Raumebenen machen es schwierig, die »Evidenz des räumlichen Arguments« (121) und damit das Modell der Jurisdiktionsökonomie in einem größeren Ordnungs- und Raumzusammenhang anzuwenden. Schade ist auch, dass eine ernsthafte Beschäftigung mit der »Komplexität des Systems« bzw. dem "Gewirr der Strukturen« (43) unter Beachtung des »exterritorialen Überbaus«, wie den Verfahren beim Reichskammergericht und dem Reichshofrat sowie der Einbindung der Fürstbischöfe in die bischöflich-päpstliche Hierarchie, nicht unternommen wurde. Weiterhin ist das Wir-

\section{Johannes Liebrecht Teile ohne Ganzes*}

Wie in der US-amerikanischen law and literature-Bewegung gehen seit Jahren auch hierzulande viele Juristen dem spannenden Wechselbezug von Recht und Literatur nach. In den philologischen Wissenschaften wird dem Themenbereich ebenfalls Aufmerksamkeit geschenkt, und seine Spielarten sind zahlreich. Zu glauben, dass es dabei interdisziplinär zugehen müsse, wäre jedoch Selbsttäuschung. Die beteiligten Literaturwissenschaftler interessieren sich so gut wie nie für das eigentlich rechtswissenschaftliche Bild vom betroffenen Recht, andererseits sind auch für Juristen die ken des Tribunals der Apostolischen Nuntiatur nicht in Betracht gezogen worden, die ebenso in der stark angefochtenen Zivil- und Kriminalitätsgerichtsbarkeit in zweiter bzw. dritter Instanz, also nicht ausschließlich in geistlichen Sachen (52), auf der regionalen Ebene wirken kann und für den Justiznutzer ebenso Appellationsinstanz in weltlichen Zivilsachen war. Zudem hätte die Beachtung der »römischen « Quellen dem topografischen Argument innerhalb des Jurisdiktionsmarktes zusätzliche Schärfe verleihen und damit weitere Kontaktpunkte innerhalb der norddeutschen Territorien mit ihren Verästelungen erfassen können. Überhaupt wird der Stellenwert der römischen Kurie und ihre Wirkungszusammenhänge auf der lokalen Ebene nicht deutlich. Weitere Studien eines Ineinanders von diachronen und raumübergreifenden Studien müssen nun zeigen, ob der eruierte Neuansatz nicht tatsächlich einen Sonderfall darstellt. literarischen Blickerweiterungen kaum von irgend nennenswerter, echter Relevanz. ${ }^{1}$ Das Lamento, beide Perspektiven schrieben aneinander vorbei und nähmen die je andere nie hinreichend auf, kehrt immer wieder. Dass die Erwartung von Interdisziplinarität gleichwohl weiterhin gehegt wird, irritiert dabei etwas, schließlich handelt es sich bei law as oder auch in literature nicht eigentlich um die Begegnung zweier Wissenschaften.

Einer solchen Erwartung scheint auch der von Claudia Lieb und Christoph Strosetzki herausgegebene Sammelband anzuhängen. Einerseits möchte

\footnotetext{
Claudia lieb, Christoph Strosetzki (Hg.), Philologie als Literatur- und Rechtswissenschaft. Germanistik und Romanistik 1730-1870, Heidelberg 2013, 286 S., ISBN 978-3-8253-5913-3
} 
er helfen, die große Lücke zu schließen, die in der Geschichte der modernen Rechts- und Literaturwissenschaften noch immer hinsichtlich der Wechselbeziehung beider klafft, andererseits meint er, gerade damit einen Beitrag zur »Vorgeschichte der Law and Literature-Bewegung « zu bieten, was nur in wenigen und zufälligen Ausnahmen ein zum ersten passendes Ziel sein dürfte. Wer sich aber die Mühe macht, den Wechselbezug beider Disziplinen aufzuarbeiten, wird auf interessante Einsichten stoßen, denn in den vergangenen drei Jahrhunderten teilten beide Fächer mehrere Fragestellungen. Das betrifft nicht allein die rechtshistorische Mediävistik, in der es unverändert Aufnahmen sprachgeschichtlicher Interpretamente gibt. Gerade dem Rechtshistoriker fallen weitere methodische Verwandtschaften in die Augen, so wenn er auf die aktuell stark vertretene pragmatische Sprachforschung schaut; statt einer isolierten Betrachtung von sprachhistorischer Veränderung betont sie die pragmatisch-situative Verwendung von Sprache in der Geschichte, möchte mithin den Wandel der normativen Struktur von Sprache nicht in deren eigener Formengeschichte, sondern durch den historischen Kontext ihrer gesellschaftlich-kommunikativen Praxis erklären. ${ }^{2}$ Andererseits lassen derlei Ähnlichkeiten auch stutzen, denn natürlich ist das rechtshistorische Fragen allenfalls bedingt repräsentativ für die Rechtswissenschaften schlechthin, und auch in der Philologie sieht es nicht viel anders aus: Ihre Teilgebiete der Sprach- und Literaturwissenschaften leben heute wortlos nebeneinander und koexistieren nur noch pro forma unter dem Dach, das sie einst von der Nationalphilologie des frühen 19. Jahrhunderts erbten. Offenbar ist also von einer Nähe >der Rechtswissenschaft zu >der $<$ Philologie, um die es im Sammelband gehen soll, heute nicht viel zu erzählen; freilich vertiefen sich gegenseitige Fremdheit wie auch die Auffächerungen innerhalb der Disziplinen selbst erst zum Ausklang der Epoche zwischen 1730 und 1870, die der Band allein behandeln will.
Inwiefern aber standen philologische und juristische Wissenschaft in wirklich erheblicher Nähe zueinander? Dazu erfährt der Leser erstaunlich wenig Genaues. Sicher ist im Groben vieles bekannt: die durchlässige Gelehrtenkultur des 17. und 18. Jahrhunderts, in der Artefakte der deutschen Sprache, Geschichte und Kultur häufig von Gelehrten des einheimischen Rechts aufgezeichnet wurden; der ungeheure Aufwind und die tiefen Verschiebungen für das Verstehen von Sprache wie von Recht, die sodann im Umfeld der frühromantischen Bewegung durch ihre Umbewertung der Hermeneutik ausgelöst wurden. Für den Zugriff auf Recht wie auf Sprache gleichermaßen wirkte sich die vertiefte Fixierung auf das Altdeutsche bei den Gebrüdern Grimm aus, die offenbar unter starkem Eindruck des Denkens Friedrich und August Wilhelm Schlegels oder Ludwig Tiecks standen. ${ }^{3}$ Die Poesie enthalte das Recht gleich wie das Gesetz die Poesie in sich schlösse, lehrte Jakob Grimm, ${ }^{4}$ und um 1840 entlehnte die philologische Germanistik gar ihre Bezeichnung von der heute eher als Namensmumie fortgeschleppten juristischen Schwester. ${ }^{5}$ Schon ob Grimm selbst zuvor wirklich unter signifikantem "Einfluss" eines vermeintlichen juristischen Lehrers Savigny gestanden hat, ist jedoch alles andere als klar. ${ }^{6}$ Ohnehin gibt es zur Lage des früheren 19. Jahrhunderts nur wenige eingehende Spezialstudien, zumal alles Übrige betreffend fehlt derzeit jede Übersicht. ${ }^{7}$

Die komparative Erfassung zweier sich wandelnder Wissenschaftskulturen und die Bewertung ihrer jeweiligen Wechselbezüge setzen freilich ein Analyseraster voraus, das kein Sammelband durchführen kann. Etwas erstaunlich wirkt aber, dass nur eine Minderzahl der Beiträge sich überhaupt mit dem Oberthema des Bandes, der Wechselwirkung zweier sich formierender Wissenschaften, beschäftigt. Nur rudimentäre Bezüge darauf finden sich bei Sigrid G. Köhlers Darstellung zu Adam Müller, wenngleich der Jurist Savigny erwähnt und, wenig überraschend, ein vertragstheoretisches Moment auch in Müllers Schriften aus-

2 Als Überblick Cherubim (1998).

3 Klausnitzer (2001) 517-519.

4 Grimm (1882) 154.

5 Meves (1994).

6 Wyss (1979) 54-93.

7 Das Opus magnum von SchäFER (2008) hat eine andere Ausrichtung; nur wenig enthält STRIPPEL (1974). 
gemacht wird (205-222), oder bei Kaspar Renner, der mit Viehweg und Curtius das Dreiecksverhältnis von Philologie, Jurisprudenz und Geschichte neu bestimmen möchte (223-245). Ebenso widmet sich dem Leitthema nur bedingt Christopher F. Laferls aus rechtshistorischer Perspektive hochinteressante Darstellung über die Kritik an der sprachlichen Fassung der um 1900 anstehenden brasilianischen Zivilrechtskodifikation, mit der Rui Barbosa den gesetzgeberischen Fortgang über 10 Jahre hinweg lahmzulegen vermochte (273-286), so interessant sich diese Episode von law as literature liest, gerade im Vergleich zur etwa zeitgleichen germanistisch-ideologischen Kritik an der vermeintlich misslungenen Sprache des BGB. Als genuin rechtshistorischer Beitrag lässt sich auch Thomas Weitins Untersuchung lesen, in der die Rolle der Genie-Idee für die gedankliche Etablierung des Urheberrechts als eigentümliches und Eigentums-Recht nachvollzogen wird (73-91), ebenfalls Stefan Willers Referat zu Diskussionen des 19. Jahrhunderts um das Urheberrecht an Schillers Werken und Lachmanns Editionen (93-107) sowie Walter-Bruno Bergs Darstellung der Argumente und Strategien, mit denen 1857 die berühmten französischen Immoralismus-Prozesse gegen Flaubert und Baudelaire juristisch ausgefochten wurden, ein Beispiel für literature in lawsuits (259-272). Wissenschaftsgeschichtlich geht hingegen Hans-Peter Haferkamp vor, der die Deutungen der Rezeption römischen Rechts in der Rechtswissenschaft des 19. Jahrhunderts vorstellt (247-258). Ähnlich wie er mit nur flüchtigem Blick für die andere Seite kommt Christian Pietsch aus, der August Boeckhs Konzept von Philologie als Grundlagenerkenntnis rekonstruiert (53-71), weitgehend auch Christoph Strosetzki, der das verflochtene Verhältnis von germanistischer und romanistischer Philologie im früheren 19. Jahrhundert darstellt (127-142). Verbindungen zeichnen sich deutlicher in der philosophie- und begriffsgeschichtlichen Interpretation Eric Achermanns zur Verbindlichkeit von Sprache und Recht insbesondere in den Schriften Pufendorfs ab (13-36), auch bei Gerda Hassler, die den Volksgeistkonzepten nachgeht, die zu Sprache und Recht im 18. Jahrhundert von Mitgliedern der Berliner Akademie vorgetragen wurden (37-51). Am stärksten bringt sie Dietrich Briesemeisters spannende Diskursgeschichte über die Beschäftigung mit dem gotischen Ursprung Spaniens zur Geltung, in der viele genuin rechts-, verfassungsund sprachhistoriographisch verwobene Interessen der romanistischen Mediävistik zu erkennen sind (109-126). Auch in verbindender Perspektive sind Passagen von Claudia Lieb verfasst, die einige Rezeptionen des berühmten Reynke de vos-Epos untersucht und insbesondere auf Heineccius und Dreyer eingeht (149-159); beide Seiten beleuchtet schließlich Joachim Rückert, der detektivisch nach möglichen Anregungen durch philologisches Denken vor und im Umfeld von Friedrich Carl v. Savignys Neuerfindung der rechtwissenschaftlichen Methode sucht (185-204). Trotz der bekannten Nähe Savignys zur philologischen Denkform und der unübersehbar zentralen Rolle, die diese in seiner wissenschaftlichen Konzeption einnahm, sind aber spezifische, klar erkennbare Vorbildkonzepte nicht zu identifizieren (202-204).

Die Herausgeber enthalten sich jedes Versuchs, die Beiträge und Ansätze ihres Sammelbandes in ein Verhältnis zueinander zu setzen. In einer unverbindlichen Skizze geben sie ein lose anreißendes brain storming über manche der angesprochenen Themen, und ihr resümierender Schlussabsatz ist so luftig, dass auch sperrige Fragen hindurch fallen. Das ist schade, denn ihre Fragestellung wirtt etliche unerschlossene Probleme des Kontakts zweier sich modernisierender Disziplinen auf, die einer Problematisierung wert wären. Wurde mit der Hinwendung der Philologie zu exakten Methoden die semantische Verschmelzung beider Wissensfelder tatsächlich, wie Hassler nahelegt (51), zunehmend obsolet? Dafür spricht einiges wo und wie weit aber trug dann der romantische Mythos der Verflochtenheit? Auf den Germanistenversammlungen wurde die berufene große $\mathrm{Ge}$ meinsamkeit keineswegs immer gelebt, ${ }^{8}$ und bei der Idee von der Philologie als Leitwissenschaft hat es sich offenbar auch um die Selbstmystifizierung einer sich etablierenden Disziplin gehandelt (Strosetzki, 129). Savigny mindestens legte sich seine philologische Orientierung eigenständig und unabhängig zu (Rückert, 202), und später brachte ein Jurist wie Theodor Mommsen dem philologischen Handwerk fast Arroganz entgegen; gegenüber einem kümmerlichen Dämmern der Philologen war bei ihm allein der im klaren Licht wandelnde 
Rechtswissenschaftler zum eigentlich höheren Verständnis berufen. ${ }^{9}$ In der blühenden Rechtswissenschaft des 19. Jahrhunderts waren Edieren und textkritisches Können zwar Basiskompetenzen, ähnlich wie es unter Gesellschaftsrechtlern heute gilt, ökonomische Grundbegriffe zu beherrschen; Karl Zeumers Karriere an der Berliner Juristischen Fakultät oder die zahllosen bekannten Editionen illustrieren dies. Aber es ist noch unerfasst, in welcher Weise die wissenschaftliche Jurisprudenz darüber hinaus erkennbar von der philologischen Disziplin beeindruckt war. $\mathrm{Ob}$ andererseits philologische Gelehrte nennenswert am nicht erst im 19. Jahrhundert suspekten, zudem sich wandelnden Terrain der Rechtswissenschaft interessiert waren, ist noch weitaus schlechter zu erkennen. Konzeptionell dürften sie wenig betroffen gewesen sein, und nur in eher kleiner Dosis tauchen Bereiche geteilter Fragen und Neugierde auf, wie das verfassungshistorische Interesse bei Boeckh oder später Scherer und anderen. Offenbar waren die Grundlage für Schnittmengen hier wie da vor allem die genuin historischen Fragestellungen, die sodann spätestens seit Mitte des 19. Jahrhunderts für beide Disziplinen im Ganzen mehr und mehr an Gewicht zu verlieren begannen. Die gelebte Nachbarschaft von wissenschaftlicher Philologie und Jurisprudenz war dann vor allem eine von Sprach- sowie Textgeschichte und Rechtsgeschichte, ${ }^{\mathbf{1 0}}$ also eine Beziehung vermittelt über das historische Paradigma. Nicht zufällig gilt gerade das philologische Interesse innerhalb der historischen Rechtswissenschaft als treibende Kraft, die rechtshistoriographische Denkform von der Wissenschaft des geltenden Rechts zu emanzipieren, ${ }^{\mathbf{1 1}}$ und auch durch die verschiedenen Entstehungsstadien der Philologie hindurch scheint es, als sei ihre Verflechtung mit der gelehrten Jurisprudenz diejenige mit und in der gemeinsamen Nationalhistorie und deren Mythen gewesen. Noch zum Ende der im Buch thematisierten Epoche erlebte diese einen erneuten Aufschwung. So oft hingegen dieser Grund fehlte, hatte dies Folgen für die Berührungen beider Fächer. Ihre Verbindung im Bereich der romanistischen Wissenschaften etwa, die der Band einbegreifen möchte, hätte ein ganz anderes Tableau zu ergeben. Bei der juristischen und der philologischen Romanistik handelt es sich eher um zwei faux amis der Wissenschaftsgeschichte, das meistenteils komparatistische Vorgehen der entstehenden philologischen Romanistik steht einem noch stark substantialistischen Denken in der eingesessenen juristischen gegenüber. Die schmale Gemeinsamkeit, sich auf in Latein verfasste, im weitesten Sinne auf antike Kultur zurückgehende Texte zu beziehen, dürfte zu wenig sein, um wie in den Germanistiken signifikante Parallelen zu vermuten. $\mathrm{Ob}$ es irgend tragfähigere Brücken gab, erfährt der Leser nicht.

Jenseits der auffallenden historiographischen Verklammerung waren beide Fächer natürlich immer, gemeinsam mit der Theologie, über die Frage nach der zutreffenden Methode für Textauslegung verschränkt, und hier lässt sich etlichen weiteren Fragen nachgehen. ${ }^{\mathbf{1 2}}$ Beide Kopplungen, in der Exegese von Texten wie in der Auslegung und Beschreibung von Vergangenheit, dürften zwar variabel und in praxi meist ineinander verlaufen sein. Sie scheinen aber auf Strukturen und Denkformen $\mathrm{zu}$ beruhen, die sich schon vor der Ausund Binnendifferenzierung beider Fächer im 19. Jahrhundert abzuzeichnen begannen und nach denen näher abzuschichten und strukturiert zu fragen sich lohnen könnte. Auch in eher institutioneller Hinsicht lassen sich Vergleichungen vertiefen: den offenbar je unterschiedlichen Einfluss von Praktikern auf den wissenschaftlichen Prozess, den Lehrern hier und der Richterschaft da; die zentrale Rolle wissenschaftlicher Generaldebatten, des Nibelungenstreits hier und der Germanisten/ Romanisten-Kontroverse dort, beide ganz unterschiedlichen Zuschnitts, doch von offenbar ähnlich treibender Wirkung für die weitere Ausdifferenzierung; die auffallende Gründung wissen-

9 Mommsen (1885) 263.

10 SCHMidT-WIEGAND (1998); vgl. auch kürzlich Fruscione (2005).

11 OgOReK (1994) 16-19.

12 Über Parallelen Savignys zu Schleiermachers Verstehenslehre RüCKERT (2001) 310-324, 326; zur zentralen Rolle Friedrich Schlegels BeIsLER (2001) $247 \mathrm{f}$. 
schaftlicher Organe gegen Ende des Untersuchungszeitraumes. Solche und ähnliche Fragen könnten künftig intensivere Bearbeitung finden, mögen sie sich auch mit der Idee einer Vorgeschichte zu law and literature schlechter vertragen.
Vielleicht wird dann hinter vielen Facetten und mancher glitzertheoretischen Pirouette ein Bild besser zu erahnen sein.

\section{Bibliographie}

- Beisler, Hermann, Die Unergründlichkeit des Werks und die Unendlichkeit der Interpretation, in: SchröDER (2001) 217-248

- Besch, Werner u.a. (Hg.) (1998), Sprachgeschichte. Ein Handbuch zur Geschichte der deutschen Sprache und ihrer Erforschung, 2. Aufl. Berlin

- Cherubim, Dieter (1998), Sprachgeschichte im Zeichen der linguistischen Pragmatik, in: Besch u. a. (Hg.) (1998) 538-550

- Fruscione, Daniela (2005), Zur Frage eines germanischen Rechtswortschatzes, in: ZRG GA 122, 1-41

- Grimm, Јасов (1882), Von der Poesie im Recht [1815], in: Ders., Kleinere Schriften, Bd. VI, Berlin, 152-191

- Klausnitzer, Ralph (2001), „Verschwörung der Gelehrten«? Die Brüder Grimm und die Romantik, in: Zeitschrift für Germanistik NF 11, 513-537

- Meves, Uwe (1994), Zur Namensgebung `Germanist`, in: Fohrmann, Jürgen, Wilhelm Vosskamp (Hg.), Wissenschaftsgeschichte der Germanistik im 19. Jahrhundert, Stuttgart, 25-47

- Mommsen, Theodor (1885), Bürgerlicher und peregrinischer Freiheitsschutz im römischen Staat, in: Juristische Abhandlungen. Festgabe für Georg Beseler, Berlin, 253-272

- Ogorek, Regina (1994), Rechtsgeschichte in der Bundesrepublik (1945-1990), in: Simon, Dieter (Hg.), Rechtswissenschaft in der Bonner Republik, Frankfurt, 12-99

- Posner, Richard A. (1988), Law and Literature. A Misunderstood Relation, Cambridge Mass.

- Rückert, Joachim (2001), Savignys Hermeneutik - Kernstück einer Jurisprudenz ohne Pathologie, in: SchröDer (2001) 287-327

- Schäfer, Frank Ludwig (2008), Juristische Germanistik, Frankfurt a. M.

- Schmidt-Wiegand, Ruth (1998), Deutsche Sprachgeschichte und Rechtsgeschichte bis zum Ende des Mittelalters, in: Besch u. a. (Hg.) (1998) 72-87

- SchröDer, Jan (Hg.) (2001), Theorie der Interpretation vom Humanismus bis zur Romantik - Rechtswissenschaft, Philosophie, Theologie. Beiträge zu einem interdisziplinären Symposion, Stuttgart

- Strippel, Jutta (1974), Zum Verhältnis von Deutscher Rechtsgeschichte und Deutscher Philologie, in: Müller, Jochen Jörg (Hg.), Germanistik und deutsche Nation 1806-1848. Zur Konstitution bürgerlichen Bewußtseins, Stuttgart, 113-166

- Weimar, Klaus (2003), Geschichte der deutschen Literaturwissenschaft bis zum Ende des 19. Jahrhunderts, Paderborn

- Wyss, Ulrich (1979), Die wilde Philologie. Jacob Grimm und der Historismus, München

\section{Santiago Legarre \\ Fortalezas y debilidades en el estudio del constitucionalismo latinoamericano*}

Como sucede con una persona, así también con el libro de Roberto Gargarella que tengo el honor de comentar: sus fortalezas son también sus debilidades. En estas líneas incompletas, intentaré fundamentar esta sentencia, no sin antes animar vivamente al lector de esta reseña a leer también la obra.
Llama la atención un proyecto que, ya desde su mismo título, pretende abarcar el derecho constitucional latinoamericano durante un período de doscientos años. ¿Acaso existe tal cosa como un «derecho constitucional latinoamericano»? (La pregunta no es del todo distinta, dicho sea de paso, de otras afines: ¿tiene sentido hablar de un «derecho

Roberto Gargarella, Latin American Constitutionalism, 1810-2010: The Engine Room of the Constitution, Oxford: Oxford University Press 2013, 283 p., ISBN 978-0-19-993796-7 